\title{
Challenges faced by the modern bioanalytical laboratory
}

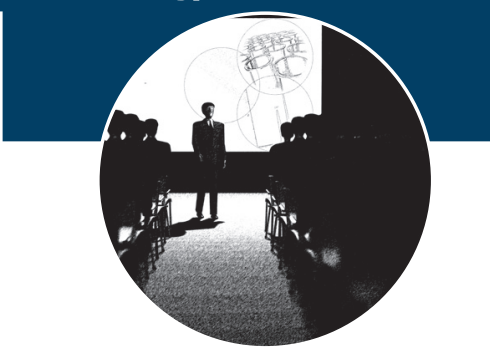

Timothy Sangster (Charles River Laboratories) and Mike Oliver (Thermo Fisher Scientific) speak to Thomas Payne at Bioanalysis in September 2012 about the challenges faced by the modern bioanalytical laboratory.

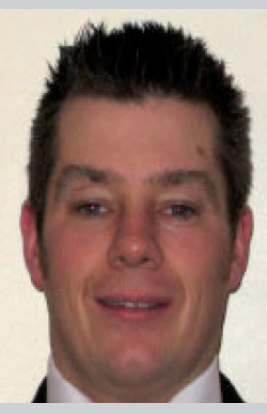

Timothy Sangster has been with Charles River Laboratories since September 2009. Having worked for Quintiles, Pharmacia, Astrazeneca and Huntingdon Life Sciences, he has gained experience over 17 years in both CRO and pharmaceutical environments, and also in both Europe and the USA. Specific areas of interest over the past years have been microchromatography, sample preparation and matrix effects, to name a few.

Mike Oliver has held the position of Global Product Manager for sample preparation at Thermo Fisher Scientific since 2010, being responsible for the development and introduction of new innovative technologies such as SOLATM to the market place. Prior to this role, Mike has worked for two leading MS vendors over a 9-year period, being responsible for biotechnology sales within the UK and providing application solutions for proteomic and metabolic workflows based on high-resolution LC-MS platforms, respectively. Mike holds a PhD in MS and Biochemistry from the MS Research Unit, University of Wales, Swansea, UK.

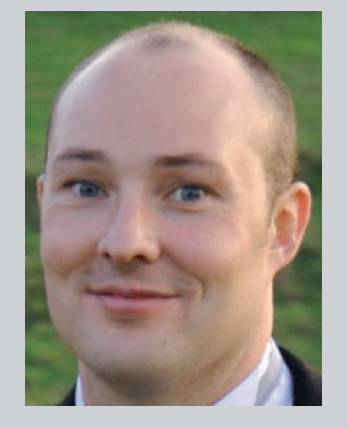

What are the challenges and demands currently faced by the modern bioanalytical laboratory?

Timothy Sangster (TS): As the industry is changing we face many challenges, and to support that I suppose we are looking for the holy grail - faster, cheaper and better. That is, less time per sample, faster method development and also speed gained through more robust methods. Cheaper by taking less working time to produce the data and using less instrument time, therefore reducing the number of required instruments. Better by generating more robust data but also producing more sensitive methods from lower sample volumes, which can be utilized to improve studies by using less animals and also generating whole profiles from single animals including small rodents.

Mike Oliver (MO): Tim, this is exactly the type of feedback that we have received from many of our customers within the bioanalytical environment. They face these challenges, and these are driven by the need for more cost-effective analysis whilst maintaining high-quality results. However, what we have noticed is that customers see the financial advantages of utilizing better sample preparation to ensure that they have lower failure rates, more robust methods and improved sensitivity, resulting in lower cost per samples analysis.

What areas are key to providing a solution to these requirements?

TS: Good methods are the starting point, which begins with the sample preparation to the separation science and then the detector. We have focused very much on doing the appropriate sample preparation, and apply a lot of SPE to give the best possible sample clean-up and then follow that up by using HPLC with high levels of separation efficiency. We employ a lot of new technology including SOLATM SPE and solid-core columns.

MO: Tim has made some excellent points. The research we have conducted at Thermo Fisher Scientific has demonstrated that most bioanalytical methods are designed around the isolation and quantification of the test analytes, and with little consideration of the matrix components and what effect they can have on the performance of the assay. This work has also shown that inappropriate sample preparation techniques can actually result in a build up of residual matrix on the analytical system, which affects not just one sample, but subsequent samples within the analytical batch. This often

\section{Timothy Sangster*1} \& Mike Oliver ${ }^{2}$

'Bioanalysis \& Immunology, Charles River Laboratories, Tranent, Edinburgh, EH33 2NE, UK ${ }^{2}$ Chromatography \& MS Division, Thermo Fisher Scientific, Tudor Road, Manor Park, Runcorn, Cheshire, WA7 ITA, UK

*Author for correspondence: E-mail: timothy.sangster@crl.com 
results in situations where the assay appears to perform well most of the time but falls over in an unpredictable manner.

Q Within bioanalysis why is sample preparation required?

TS: The biologic samples that we are working with, be it blood, plasma, brain or any other biological matrix, are a very complex mixture of components. Generally, we are looking to analyze extremely low levels of one or more analytes of interest. Therefore, we have to simplify the sample, while retaining the analytes, to allow robust analysis. In many cases, we are looking to selectively extract the analyte and concentrate it while removing the rest of the matrix components to allow extremely low-level detection. The removal of unwanted matrix components reduces the background interferences in the assay and also reduces the potential for these interferences to cause significant issues, especially when using MS detection.

MO: The introduction of LC-MS to bioanalytical laboratories was seen as a huge advance in detection capability and selectivity. The sensitivity and specificity of the technique was seen as a panacea. For the first few years of its routine use in bioanalytical laboratories, there was a belief that complex sample preparation and chromatography were not required. This was shown not always to be the case when dealing with lowlevel analysis from complex matrices due to the effects of ion suppression, which are prevalent in atmospheric ionization sources. For these reasons, the need for good sample preparation and chromatography to ensure good quality data became apparent.

What does a bioanalyst want or require from their sample preparation technique?

TS: Ease of use is a critical parameter and over the past 15 years the introduction of automated SPE and the use of polymeric phases have been instrumental in the robustness of SPE. Reproducibility is now the key to guaranteeing a product can be reproduced well-to-well, plate-to-plate and batch-to-batch, as well as day-to-day, month-to-month and year-to-year. On top of this, your sample preparation technique should allow you to selectively capture the analytes of interest whilst actively removing the interferences.

MO: SPE hardware has not changed significantly over the last 20 years (loose-packed material positioned between two frits). As a result, analysts have had to cope with analytical variability due to bed inconsistencies caused by packing discrepancies and bed instability (caused during production or transportation), which can lead to voiding, channeling and even loss of stationary phase, and ultimately in a lack of robustness. Solving these issues has focused our research activities when developing innovative SPE technology, such as SOLA, which overcomes these problems and results in greater robustness and ease of use. Coupled to this is the availability of a range of different chemistries that allow the analyst choice when optimizing the extraction process to best remove interferences and selectively capture the analyte of interest.

\section{What technologies are currently} employed to facilitate sample preparation within bioanalysis?

TS: The main techniques of sample preparation routinely employed for bioanalysis are SPE, liquid-liquid extraction (LLE) and protein precipitation (PPT). The are a few others that are not as mainstream but are continuing to gain popularity including, supported liquid extraction, online techniques, which includes turbulent flow and online SPE, and recently even the use of immunoaffinity techniques are starting to become more common, using either columns or bead-based techniques.

MO: The points that Tim has made are very good. Although SPE does have a wide range of selectivity's, it is important for the bioanalyst to have a wide range of analytical techniques at their disposal, since SPE may not be applicable in all cases, and indeed the research we have been performing at Thermo Scientific has demonstrated that for certain compounds different approaches other than SPE provide optimal performance due to either compound stability or solubility issues.

\section{Q How do these technologies currently meet the requirement of the bioanalyst?}

TS: PPT is a great technique if you can afford to live with the limitations in sample clean-up, which for a lot of early-stage work is more than acceptable. Implementation of automation using PPT plates that have additional functionality to selectively remove specific interferences is a step forward and also allows easier automation. However, they still have limitations on 
the separation that need to be achieved. LLE can be a very good technique if the assays are appropriately set-up, but it is very labor intensive and time consuming. I remember working 10-h days doing a single batch of LLE samples and it was hard work. Supported liquid extraction has allowed for miniaturization and automation of this technique making it far easier to implement. SPE is, in my view, the best sample clean-up in terms of automation and separation, but comes at a cost. The technique can be significantly more expensive when compared with PPT or LLE and the optimization of the methods can take longer (although probably should not!). SPE gives the best opportunity for sample clean-up and concentration, and if done correctly will give the most robust assays that achieve the lowest limits of quantification.

MO: Tim again makes some good points. There are a number of options for sample clean-up and choice of the one that fits the analysis best is critical to the success of the assay. In some cases, whilst clean-up is not necessarily optimal, PPT can be the best choice, owing to its ease of use and speed. For an assay requiring ultimate sensitivity and reproducibility, SPE would be the most appropriate technique.

Why do these technologies currently fail to meet the desired standard for the bioanalyst?

TS: Normally, assays fall down due to the unknown, for example, we get a matrix effect in samples that was not observed during assay development or validation. This is commonly observed when using PPT, but can often be avoided when using SPE. We anticipate that a certain percentage of batches will not meet acceptance criteria and we put this down to the difficulty of the samples that are being handled. However, working with more robust technology enables us to drive this percentage down and improve the overall robustness of the assay. Automation is still a major limitation and while we can semi-automate the extraction, especially with SPE, this often presents a major challenge. These issues are normally linked to the hardware rather than the technique, for example, opening tubes, reading labels and handling poor samples (e.g., dealing with blood clots). As we are moving to new analyte classes, especially peptides and proteins, the problems we face are very different to small-molecule analytes and we are being challenged to develop new, more appropriate sample preparation techniques.
MO: Anticipating where the next analytical challenges will be is a major focus for us. We see the step change in reproducibility requirements and the move towards lower sample volumes, lower limits of detection and more complex molecules as the major areas of interest over the next few years. SOLA's unique technology is already providing considerable benefits with higher levels of reproducibility combined with the ability to provide lower elution volumes.

Why are these factors so critical to the workflow and are these demands likely to become more important in the future?

TS: Timelines are becoming more important every day, everyone wants to go faster and this puts stress on the bioanalytical workflow. Improvements in robustness allow us to be more accurate in our planning, as we need less contingency, and produce data faster to meet customer demands. The current drive to reduce sample volume has lead to a move towards dried blood spots (DBS) and capillary microsampling, which allows the bioanalyst to use a much smaller sample to generate the same high-quality data. The use of more rigorous sample clean-up and highsensitivity methods are crucial in allowing this to happen. Data quality is another major driver and the introduction of incurred sample reanalysis has lead to a greater focus on this area. The only way to reduce risk when you analyze your study samples and guarantee that you are not going to have an issue with the reproducibility is to do as much separation as possible (sample preparation and chromatography) and remove as many unknowns as possible.

MO: Tim makes two very salient points relating to assay robustness and sample volume. These are important issues that the modern bioanalyst faces on a day-to-day basis. These can be addressed by using robust technologies that are applicable to smaller sample volumes. SPE technologies have moved some way to providing a solution, however issues with the inherent design of loose-packed SPE media can result in variability in reproducibility of the assay.

\section{Q Is there anything currently available that} addresses these needs?

TS: Sample preparation is always moving forward, be it with the introduction of polymeric materials for SPE or to the newer materials that are giving better separation and control of the sample clean-up process, for example, SOLA. 
Chromatography is also an ever-changing landscape and over the last 10 years we have seen the advent of ultra-HPLC, small particle sizes and, more recently, solid-core particles (e.g., Accucore ${ }^{\mathrm{TM}}$ ).

MO: The technology associated with SOLA SPE ensures that sample-to-sample robustness is assured, while also providing lower elution volumes and thus the use of lower sample volumes. This is achieved by combining the polymeric frit material and stationary phase into a single entity, rather than an assembled form consistent of powder and separate frits as in conventional loose-packed SPE. The conventional SPE format is prone to voiding, channeling and inconsistencies in packing, which is accentuated at lower bed weights. The manufacturing process associated with SOLA's unique design ensures that consistent bed weight and bed size are achieved even when subjected with physical stresses. This results in providing a solution that addresses the issues which Tim mentions.

What impact has this new technology on the bioanalytical workflow?

TS: We have been able to develop more robust methods in a shorter time. The analysis is also completed in a shorter time and with less problems. We can increase sensitivity using new SPE, HPLC and detector technology, and this allows us to implement both DBS and capillary microsampling samples within our company, enabling refined and reduced animal use.

MO: The comments that Tim makes are very encouraging. Whilst developing this new technology we have conducted extensive in-house testing, which supports Tim's experiences within a leading high-throughput bioanalytical laboratory. From feedback from our customers, they are seeing a significant change in performance, robustness and quality of their results since implementing this novel technology.

Q Do you ever envisage a time when advancements in LC-MS instrumentation will remove the need for sample preparation? For example, direct analysis, especially in conjugation with MS and tissue bioanalysis, shows promising potential, but, in your opinion, will some sort of sample preparation/extraction always be required?

TS: While in some situations the use of direct analysis is feasible, bioanalysis will always be subject to the limitations of matrix effects.
So for quantification in a regulated environment, the use of some form of sample cleanup will be required for the foreseeable future. In qualitative work, the use of high-resolution instrumentation (or other developments) may allow analysis with limited or no separation, but the risks of slight changes in the sample matrix impacting any quantification will, for the meantime, result in us continuing to do significant amounts of separation chemistry. During the advances over the past 20 years, there have been a couple of times when the death of sample preparation has been heralded only for us to then find many reasons why we should continue to do good, fit-for-purpose sample clean-up prior to analysis.

MO: I agree with Tim's statement. Sample preparation not only provides reduced interferences within MS, but also reduces the amount of maintenance associated with a particular assay. Finally, the use of sample preparation allows for preconcentration of the sample, which provides increased sensitivity. Whilst a radical new design in the source of a MS may reduce the amount of ion suppression, this will not address other issues associated with contamination of the sampling device or the requirement for the development of more sensitive assays.

What do you see as the future for sample preparation within bioanalysis? New innovations are continually being developed, but what sample preparation/extraction technology would you like to see in the future?

TS: Continued developments in handling smaller sample volumes in well plate or tip format are important. These techniques need to be backed up by consumables for handling the sample, from collection to analysis, in a 96 well or higher format. I believe these have not become main stream techniques due to limitations in the format and ease of use. Automation and software that can handle the whole process from sample arrival to reported sample results, encompassing automated sample preparation, sample analysis and data reporting, is crucial. Large molecules are a 'relatively' new area for chromatographicbased regulated bioanalyitcal methods, especially with a MS end point. Developments in this area to support the process would be nice to see. Easier integration of immunoaffinity analysis into the workflow, so that we can utilize this more easily and cost effectively would also be a nice to have. 


\section{INTERVIEW I NEWS \& ANALYSIS}

MO: There are three key areas for future development of sample preparation. As Tim has alluded to, more selective phases will ensure less matrix effects are observed, providing the bioanalyst with more robust assays. The development of the biopharmaceutical industry has resulted in many new challenges in the field of bioanalysis. Large biomolecules are incredibly complex and ensuring that the sample preparation is selective will be a major challenge for the industry. Finally, there is the question of DBS, and the hematocrit issue, which requires a solution.

'Green' bioanalysis and bioanalytical chemistry is of growing importance, but what are the main implications of this in sample preparation and extraction?

TS: Reduction of solvent usage is probably the biggest driver, and this can be achieved by using low bed volume SPE. This also then follows through to the HPLC, if you run at $100 \mu \mathrm{l} / \mathrm{min}$ instead of $1 \mathrm{ml} / \mathrm{min}$ you can reduce solvent use by tenfold. I remember when the first dedicated online SPE systems were released and we were told that you could reuse the cartridge 20-times! Can we come up with a SPE plate we can reuse to reduce wastage and decrease costs but without any risks?

MO: With the advent of new sample preparation technologies there has been a reduction in the requirements for organic solvent. As the industry drives towards even lower sample volumes, manufactures will be tasked to provide solutions that will address these challenges. This will result in greener assays and analysis.

Financial \& competing interests disclosure $M$ Oliver works on behalf of Thermo Fisher Scientific, who own the brand SOLATM. The authors have no other relevant affiliations or financial involvement with any organization or entity with a financial interest in or financial conflict with the subject matter or materials discussed in the manuscript apart from those disclosed.

No writing assistance was utilized in the production of this manuscript. 